\title{
PROXIMAL EXTRAGRADIENT METHODS FOR PSEUDOMONOTONE VARIATIONAL INEQUALITIES
}

\author{
MUHAMMAD ASLAM NOOR ${ }^{1}$ AND ABDELLAH BNOUHACHEM ${ }^{2}$
}

\begin{abstract}
We consider and analyze some new proximal extragradient type methods for solving variational inequalities. The modified methods converge for pseudomonotone operators, which is a weaker condition than monotonicity. These new iterative methods include the projection, extragradient and proximal methods as special cases.
\end{abstract}

\section{Introduction}

Variational inequalities have had a great impact and influence in the development of almost all branches of pure and applied sciences. There are several numerical methods including projection, the Wiener-Hopf equations, proximal auxiliary principle techniques for solving variational inequalities, see [1-6]. It is well known that the convergence of the projection method requires the operator $T$ to be strongly monotone and Lipschitz continuous. These strict conditions rule out many applications of the projection method for a wide class of problems. These facts motivated to modify the projection method and its variant forms. The extragradient method overcomes this difficulty by the technique of updating the solution, which modified the projection method by performing additional step and projection at each step according to double projection formula. It is worth mentioning that the convergence of the extragradient method requires that the solution exists and the operator to be monotone and Lipschitz continuous. When the operator is not Lipschitz continuous or when the Lipschitz continuous is not known, the extragradient method and its variant forms require an Armijo-like line search procedure to compute the step size with a new projection needed for each trial, which leads to expensive computation. To overcome these draw backs, many authors have suggested and proposed some modified extragradient methods for solving variational inequalities. Recently He at el. [4] have considered a class of modified proximal-extragradient methods, which uses a

Received and revised October 8, 2004.

2000 Mathematics Subject Classification. 49J40, 90C30.

Key words and phrases. Variational inequalities, projection method, fixed point, inertial proximal methods, convergence.

${ }^{1}$ This author is supported by the Higher Education Commission, Pakistan, through research grant No: 1-28/HEC/HRD/2005/90.

${ }^{2}$ This author was supported by NSFC grant 10571083 . 
better step-size rule (inexactness criteria) and includes the proximal and the extragradient methods as a special cases. They have shown the convergence of this approximate proximal method requires only monotonicity. Inspired and motivated by the research going in this dynamic field, we suggest a new modified proximal extragradient method for solving the variational inequalities. We show that the convergence of our methods requires the pseudomonotonicity. As a special case, we conclude that the convergence of the approximate proximal extragradient method of He, Yang and Yuan [4] requires the pseudomonotonicity, which is a weaker condition than monotonicity. Thus our results improve the convergence criteria of methods of He and Yang [4]. Our results can also be viewed as a significant extension and generalization of the previously known methods for solving variational inequalities and related optimization problems.

\section{Formulation}

Let $H$ be a real Hilbert space, whose inner product and norm are denoted by $\langle\cdot, \cdot\rangle$ and $\|\cdot\|$ respectively. Let $K$ be a closed convex set in $H$ and $T: H \rightarrow H$ be a nonlinear operator. We consider the problem of finding $u \in K$ such that

$$
\langle T u, v-u\rangle \geq 0, \quad \forall \quad v \in K .
$$

Problem (1) is called the variational inequality, which was introduced and studied by Stampacchia [8] in 1964. It has been shown that a large class of obstacle, unilateral, contact, free, moving, and equilibrium problems arising in regional, physical, mathematical, engineering and applied sciences can be studied in the unified and general framework of the variational inequalities (1), see [1-12].

Lemma 2.1. For a given $z \in H, u \in K$ satisfies the inequality

$$
\langle u-z, v-u\rangle \geq 0, \quad \forall v \in K
$$

if and only if

$$
u=P_{K}[z]
$$

where $P_{K}$ is the projection of $H$ onto $K$. Also, the projection operator $P_{K}$ is nonexpansive and satisfies the inequality

$$
\left\|P_{K}[z]-u\right\|^{2} \leq\|z-u\|^{2}-\left\|z-P_{K}[z]\right\|^{2} .
$$

Definition 2.1. $\forall u, v \in H$, the operator $T: H \rightarrow H$ is said to be (i) monotone, if

$$
\langle T u-T v, u-v\rangle \geq 0 .
$$

(ii) pseudomonotone, if

$$
\langle T u, v-u\rangle \geq 0 \quad \text { implies }\langle T v, v-u\rangle \geq 0 \text {. }
$$


Note that monotonicity implies pseudomonotonicity but the converse is not true [2].

\section{Main Results}

In this section, we use the projection technique to suggest some iterative methods for solving the variational inequalities. For this purpose, we need the following result, which can be proved by invoking Lemma 2.1 .

Lemma 3.1. The function $u \in K$ is a solution of (1) if and only if $u \in K$ satisfies the relation

$$
u=P_{K}[u-\rho T u]
$$

where $\rho>0$ is a constant.

Lemma 3.1 implies that problems (1) and (5) are equivalent. This alternative formulation is very important from the numerical analysis point of view and has played a significant part in suggesting several numerical methods for solving variational inequalities and complementarity problems, see [17,10-12].

We now define the projection residue vector by the relation

$$
\begin{aligned}
R(u) & =u-P_{K}[u-\rho T u]=u-y, \\
y & =P_{K}[u-\rho T u] .
\end{aligned}
$$

Invoking Lemma 3.1, one can easily show that $u \in K$ is a solution of (1) if and only if $u \in K$ is a zero of the equation

$$
R(u)=0 .
$$

For a positive constant $\alpha$, equation (6) can be written as:

$$
u=u-\alpha R(u)=u-\alpha\left\{u-P_{K}[u-\rho T u]\right\} .
$$

We use this fixed-point formulation to suggest the following iterative method for variational inequalities (1).

This fixed-point formulation can be used to suggest the following iterative method.

Algorithm 3.1. For a given $u_{0} \in H$, compute the approximate solution $u_{n+1}$ by the iterative scheme

$$
\begin{aligned}
u_{n+1} & =P_{K}\left[u_{n}-\gamma_{n} R\left(u_{n+1}\right)\right] \\
& =P_{K}\left[u_{n}-\gamma_{n}\left\{u_{n}-P_{K}\left[u_{n}-\rho T u_{n+1}\right]\right\}\right], \quad n=0,1,2, \ldots,
\end{aligned}
$$

or equivalently

$$
\begin{aligned}
y_{n} & =P_{K}\left[u_{n}-\rho T u_{n+1}\right] \\
u_{n+1} & =P_{K}\left[u_{n}-\gamma_{n}\left\{u_{n}-y_{n}\right\}\right], \quad n=0,1,2, \ldots
\end{aligned}
$$


which can be considered as a proximal point method and appears to be a new one. Note that for $\gamma_{n}=1$, Algorithm 3.1 reduces to:

Algorithm 3.2. For a given $u_{0} \in H$, compute the approximate solution $u_{n+1}$ by the iterative scheme

$$
u_{n+1}=P_{K}\left[u_{n}-\rho T u_{n+1}\right], \quad n=0,1,2 \ldots
$$

which is known as the proximal method. In recent years, proximal methods have been considered and studied extensively. Several conditions have been studied which are easy to implement and to accelerate the convergence; see $[4,10,12]$.

Now we look at Algorithm 3.3 from a different angle. Consider $y$ defined by (5) as an approximate solution of the variational inequality (1) and define

$$
\begin{aligned}
w & =P_{K}[u-\gamma(u-y)] \\
z & =P_{K}[u-\rho T w] .
\end{aligned}
$$

We use this formulation to suggest the following iterative method

Algorithm 3.4. For a given $u_{0} \in H$, calculate the approximate solution $u_{n+1}$ by the iterative schemes;

$$
\begin{aligned}
y_{n} & =P_{K}\left[u_{n}-\rho T u_{n}\right] \\
w_{n} & =P_{K}\left[u_{n}-\gamma\left(u_{n}-y_{n}\right)\right] \\
u_{n+1}:=z_{n} & =P_{K}\left[u_{n}-\rho T w_{n}\right], \quad n=0,1,2, \ldots
\end{aligned}
$$

which is called the modified extragradient method and appears to be a new one. Note that for $\gamma=1$, Algorithm 3.4 reduces to

Algorithm 3.5. For a given $u_{0} \in H$, compute the approximate solution $u_{n+1}$ by the iterative scheme

$$
\begin{aligned}
y_{n} & =P_{K}\left[u_{n}-\rho T u_{n}\right] \\
u_{n+1} & =P_{K}\left[u_{n}-\rho T y_{n}\right], \quad n=0,1,2, \ldots
\end{aligned}
$$

which is exactly the extragradient method for solving the general variational inequality (1).

For a positive constant $\alpha$, consider

$$
u=u-\alpha(u-z)
$$

Here the positive constant $\alpha$ can be viewed as a step length along the direction $-(u-z)$.

We use this fixed-point formulation to suggest the following iterative method. 
Algorithm 3.6. For a given $u_{0} \in H$, compute the following iterative schemes:

$$
\begin{aligned}
y_{n} & =P_{K}\left[u_{n}-\rho_{n} T u_{n}\right] \\
w_{n} & =P_{K}\left[u_{n}-\gamma_{n}\left(u_{n}-y_{n}\right)\right] \\
z_{n} & =P_{K}\left[u_{n}-\rho_{n} T w_{n}\right] \\
u_{n+1} & =P_{K}\left[u_{n}-\alpha\left(u_{n}-z_{n}\right)\right], \quad n=0,1,2, \ldots \\
\alpha & =\frac{\left\|z_{n}-w_{n}\right\|^{2}+\left\|u_{n}-z_{n}\right\|^{2}-\triangle\left(w_{n}\right)}{2\left\|u_{n}-z_{n}\right\|^{2}}
\end{aligned}
$$

where

$$
\begin{aligned}
\triangle\left(w_{n}\right) & \leq \nu\left(\left\|z_{n}-w_{n}\right\|^{2}+\left\|u_{n}-z_{n}\right\|^{2}\right), \quad \nu<1 \\
& =\nu\left\{2\left\langle w_{n}-z_{n}, w_{n}-u_{n}+\rho_{n} T w_{n}\right\rangle-\left\|w_{n}-z_{n}\right\|^{2}\right\} .
\end{aligned}
$$

Here $\triangle\left(w_{n}\right)$ is known as the inexactness criteria and can be viewed as stepsize.

For $\alpha=1$ and $z_{n}=w_{n}$, Algorithm 3.6 is exactly Algorithm 3.4. If $y=w$, then Algorithm 3.6 reduces to:

Algorithm 3.7. For a given $u_{\in} H$, compute the approximate solution $u_{n+1}$ by the iterative schemes

$$
\begin{aligned}
y_{n} & =P_{K}\left[u_{n}-\rho_{n} T u_{n}\right] \\
w_{n} & =P_{K}\left[u_{n}-\gamma\left(u_{n}-y_{n}\right)\right] \\
u_{n+1}:=z_{n} & =P_{K}\left[u_{n}-\alpha\left(u_{n}-w_{n}\right)\right], \quad n=0,1,2, \ldots \\
\alpha & =\frac{\left\|u_{n}-y_{n}\right\|^{2}+\left\|u_{n}-w_{n}\right\|^{2}-\triangle\left(y_{n}\right)}{2\left\|u_{n}-w_{n}\right\|^{2}}
\end{aligned}
$$

which is an approximate extragradient projection method for solving (1). In particular, for $\gamma=1$, the identity operator, Algorithm 3.7 is exactly the same as considered by He, Yang and Yuan [4] for solving the variational inequalities (1). If $y_{n}$ is defined by (7), then Algorithm 3.4-3.7 are called the approximate proximal extragradient methods, which are new ones. In a similar way, one can obtain several new and known algorithms as special cases of Algorithm 3.6. This shows that Algorithm 3.6 unifies several recently proposed (implicit or explicit ) algorithms for solving variational inequalities.

We now study the convergence analysis of Algorithm 3.6. The analysis is in the spirit of He, Yang and Yuan [4] and Noor [12]. To convey the idea and for the sake of completeness, we include the details.

Theorem 3.1. Let the operator $T$ be pseudomonotone. If $u \in K$ be a solution of the variational inequality (1) and $u_{n+1}$ be the approximate solution obtained from Algorithm 3.6 , then

$$
\begin{aligned}
\left\|u_{n+1}(\alpha)-u\right\|^{2} \leq & \left\|u_{n}-u\right\|^{2}-\frac{(1-\nu)^{2}}{4}\left\{\left\|u_{n}-w_{n}\right\|^{2}\right. \\
& \left.+\left\|u_{n}-z_{n}\right\|^{2}\right\} .
\end{aligned}
$$


Proof. Let $u \in K$ be a solution of (1). Then

$$
\langle T u, v-u\rangle \geq 0, \quad \forall v \in K,
$$

implies that

$$
\langle T v, v-u\rangle \geq 0
$$

since $T$ is pseudomonotone.

Taking $v=w_{n}$ in (17), we have

$$
\left\langle T w_{n}, w_{n}-u\right\rangle \geq 0
$$

which can be written as

$$
\left\langle T w_{n}, z_{n}-u\right\rangle \geq\left\langle T w_{n}, z_{n}-w_{n}\right\rangle .
$$

Taking $z=\left[u_{n}-\rho_{n} T w_{n}\right], \quad u=z_{n}$ and $v=u$ in (2), we have

$$
\left\langle u_{n}-\rho_{n} T w_{n}-z_{n}, u_{n}-u\right\rangle \geq 0,
$$

from which we have

$$
\left\langle u_{n}-z_{n}, u_{n}-u\right\rangle \geq\left\langle u_{n}-u, \rho_{n} T w_{n}\right\rangle .
$$

¿From (18) and (19), we have

$$
\left\langle u_{n}-z_{n}, z_{n}-w_{n}\right\rangle \geq\left\langle\rho_{n} T w_{n}, z_{n}-w_{n}\right\rangle .
$$

Consider

$$
\begin{aligned}
\left\|u_{n}-u\right\|^{2}- & \left\|u_{n+1}(\alpha)-u\right\|^{2}=\left\|u_{n}-u\right\|^{2} \\
& -\| P_{K}\left[u_{n}-\alpha\left(u_{n}-z_{n}\right]-P_{K}[u] \|^{2}\right. \\
\geq & \left\|u_{n}-u\right\|^{2}-\| u_{n}-u-\alpha\left(u_{n}-z_{n} \|^{2}\right. \\
= & 2 \alpha\left\langle u_{n}-u, u_{n}-z_{n}\right\rangle-\alpha^{2}\left\|u_{n}-z_{n}\right\|^{2} \\
= & 2 \alpha\left\|u_{n}-z_{n}\right\|^{2}+2 \alpha\left\langle z_{n}-u, u_{n}-z_{n}\right\rangle \\
& -\alpha^{2}\left\|u_{n}-z_{n}\right\|^{2} .
\end{aligned}
$$

Combining (15), (20) and (21), we obtain

$$
\begin{gathered}
\left\|u_{n}-u\right\|^{2}-\left\|u_{n+1}(\alpha)-u\right\|^{2} \geq \alpha\left\{\left\|z_{n}-w_{n}\right\|^{2}+\left\|u_{n}-z_{n}\right\|^{2}\right. \\
\left.-\triangle\left(w_{n}\right)\right\}-\alpha^{2}\left\|u_{n}-z_{n}\right\|^{2},
\end{gathered}
$$

which is a quadratic in $\alpha$ and has a maximum at

$$
\alpha^{*}=\frac{\left\|z_{n}-w_{n}\right\|^{2}+\left\|u_{n}-z_{n}\right\|^{2}-\triangle\left(w_{n}\right)}{2\left\|u_{n}-w_{n}\right\|^{2}} .
$$


From (15), (22) and (23), we have the required result (16).

Theorem 3.2. Let $H$ be a finite dimensional subspace. If $u \in K$ be a solution of (1) and $u_{n+1}$ be the approximate solution obtained from Algorithm 3.6, then $\lim _{n \rightarrow \infty}\left(u_{n}\right)=$ $u$.

Proof. Let $u \in H$ be a solution of (1). From (16), it follows that the sequence $\left\{\left\|u-u_{n}\right\|\right\}$ is nonincreasing and consequently $\left\{u_{n}\right\}$ is bounded. Furthermore, we have

$$
\sum_{n=1}^{\infty} \frac{(1-\nu)^{2}}{4}\left\{\left\|z_{n}-w_{n}\right\|^{2}+\left\|u_{n}-z_{n}\right\|^{2}\right\} \leq\left\|u_{0}-u\right\|^{2},
$$

which implies that

$$
\begin{aligned}
& \lim _{n \rightarrow \infty}\left\|z_{n}-w_{n}\right\|=0 \\
& \lim _{n \rightarrow \infty}\left\|u_{n}-z_{n}\right\|=0 .
\end{aligned}
$$

Thus we see that the sequences $\left\{w_{n}\right\}$ and $\left\{z_{n}\right\}$ are also bounded. Also from (24) and (25), we have

$$
\begin{aligned}
\left\|R\left(w_{n}\right)\right\| & =\left\|w_{n}-P_{K}\left[w_{n}-\rho T w_{n}\right]\right\| \\
& =\left\|w_{n}-z_{n}+z_{n}-P_{K}\left[w_{n}-\rho T w_{n}\right]\right\| \\
& \leq\left\|w_{n}-z_{n}\right\|+\left\|P_{K}\left[u_{n}-\rho T w_{n}\right]-P_{K}\left[w_{n}-\rho T w_{n}\right]\right\| \\
& \leq\left\|w_{n}-z_{n}\right\|+\left\|u_{n}-w_{n}\right\| \\
& =0 .
\end{aligned}
$$

Thus

$$
\lim _{n \rightarrow \infty} R\left(w_{n}\right)=0 .
$$

Let $\hat{u}$ be a cluster point of $\left\{w_{n}\right\}$ and the subsequence $\left\{w_{n_{i}}\right\}$ converges to $\hat{u}$. Since $R(u)$ is a continuous function of $u$, it follows that

$$
\lim _{n \rightarrow \infty} R\left(w_{n_{i}}\right)=R(\hat{u})=0,
$$

which shows that $\hat{u}$ is a solution of the variational inequality (1). From (24) and (25), we know that $\lim _{n \rightarrow \infty}\left(y_{n_{i}}\right)=\hat{u}=\lim _{n \rightarrow \infty}\left(z_{n_{i}}\right)$. Hence from (16), we have

$$
\left\|u_{n+1}-\hat{u}\right\|^{2} \leq\left\|u_{n}-\hat{u}\right\|^{2}, \quad \forall n \geq 0,
$$

which shows that the sequence $\left\{u_{n}\right\}$ converges to $\hat{u}$, the required result.

Remark 3.1. In this paper, we have suggested and analyzed a new proximal extragradient method for pseudomonotone variational inequalities and complementarity 
problems. The convergence of the new method requires only the pseudomonotonicity of the operator, which is a weaker condition than monotonicity. Since Algorithms 3.4-3.5 and 3.7 are special cases of Algorithm 3.6, Theorem 3.1 and Theorem 3.2 continue to hold for these algorithms. In this respect, our results represent a significant improvement. The comparison of this new method with the other methods is an intertesting problem for further research.

\section{References}

[1] D. P. Bertsekas and J. Tsitsiklis, Parallel and Distributed Computation : Numerical Methods, Prentice Hall, Englewood Cliffs, New Jersey, 1989.

[2] F. Giannessi and A. Maugeri, Variational Inequalities and Network Equilibrium Problems, Plenum Press, New York, NY, 1995.

[3] B. S. He and L. Z. Liao, Improvement of some projection methods for monotone nonlinear variational inequalities, Journal of Optimization Theory and applications 112(2002), 111128.

[4] B. S. He, Z. Yang and X. M. Yuan, An approximate proximal- extragradient method for monotone variational inequalities, Journal of Mathematical Analysis and Applications 300 (2005), 362-374.

[5] M. A. Noor, New Extragradient-type Methods for General Variational Inequalities, Journal of Mathematical Analysis and Applications 277(2003), 379-395.

[6] M. A. Noor, A modified extragradient method for general monotone variational inequalities, Computers and Mathematics with Applications 38(1999), 19-24.

[7] M. A. Noor, Some algorithms for general monotone mixed variational inequalities, Mathematics and Computer Modelling 29(1999), 1-9.

[8] G. Stampacchia, Formes bilineaires coercivites sue les ensembles convexes, Comptes Rendus de l'Academie des Sciences, Paris, 258(1964), 4413-4416.

[9] M. Patriksson, Nonlinear Programming and Variational Inequalities: A Unified Approach, Kluwer Academic Publishers, Dordrecht, Holland, 1998.

[10] M. V. Solodov and B. F. Svaiter, Error bounds for proximal point subproblems and associated inexact proximal point algorithm, Mathematical Programming 88(2000), 371-389.

[11] M. A. Noor, K. I. Noor and T. M. Rassias, Some aspects of variational inequalities, Journal of Computational and Applied mathematics 47(1993), 285-312.

[12] M. A. Noor, Some developments in general variational inequalities, Applied Mathematics and Computation 152(2004), 199-277.

Mathematics Department, COMSATS Institute of Information Technology, Islamabad, Pakistan.

E-mail: noormaslam@hotmail.com

School of Management Science and Engineering, Nanjing University, Nanjing, 210093, P. R. China.

E-mail: babedallah@yahoo.com 